\title{
The Influence of Self-Organized Learning Environments (SOLEs) on EFL students in a college in Oman
}

\author{
Malik Al Zakwani ${ }^{1}$, Caroline Walker-Gleaves ${ }^{2}$ \\ Newcastle University ${ }^{1,2}$, United Kingdom
}

\begin{abstract}
This paper is part of a larger project where the researchers explored English as a Foreign Language (EFL) students' experiences in learning English using Self-Organized Learning Environments (SOLEs) within the context of their English foundation year in a college setting in Oman. The purpose that guided this current paper is to investigate whether SOLE pedagogy is able to positively influence Omani EFL college students' learning beliefs and behaviours.

Using a participatory action research design, data sources included a series of diaries, semi-structured interviews, focus groups and the researchers' field notes. Interaction between the researchers and participants and among participants themselves helped to ensure the rigour of this research. Throughout the research, participants were involved in expressing their ideas and thoughts about SOLEs and in decision making.

The results indicated that SOLEs can be an effective EFL pedagogical approach. Some strengths of SOLEs that were indicated by the results include the SOLEs' ability to increase students' autonomy and their ability to motivate students.

This empirical study assists in understanding the construction of an effective English language learning environment in an under-researched international context. It also contributes to previous and ongoing studies that investigate SOLEs in different contexts and fields to explore and examine their impact on students' experiences of higher education.
\end{abstract}

\section{Introduction}

The EFL learning environment in the College where this study was located worked as an impediment to students' EFL learning. Many students in this college often found it challenging to grasp the intricacies of the English Language at the tertiary level, especially after they had pursued their primary and secondary level of education in their mother tongue. English in Omani schools is taught as a mainly theoretical subject only and taught for approximately four hours a week. The medium of instruction in governmental schools is Arabic. In practice, most Omani pupils rarely use English in schools and outside of the school setting. This approach to language learning in Omani schools has frequently affected students negatively in terms of adapting to the newly introduced and reformed English learning environment at the tertiary level where English is the medium of instruction; the sudden exposure to English at entry level to tertiary education has further added to the problem mentioned above. Evaluation documents from the institution in this study, supported by literature in this area, demonstrates that students become shy, hesitant and lack confidence in using English due to their very low level of the language. Al-Mahrooqi and Denman [1] (p. 3) contend that: 'School graduates often have limited English language abilities which negatively impact upon their success in both tertiary level English medium environments and in a national workforce that has seemingly ever-increasing demand for employee English proficiency'.

Despite the time students spend on learning different English skills in the College within their language classes, it is observed that students face difficulties in English language when that becomes the medium of teaching and learning. This consequently affects their ability to perform well in their other studies that subsequently leads to a high unnecessary withdrawal and dismissal rate every year. The main researcher worked as an English teacher for three years and then as Assistant Dean for Student Affairs in this college, and the latter role involved responsibility for all issues related to students from enrolment until graduation, dismissal or withdrawal. It is clear through counselling sessions that students have decided to withdraw due to their negative attitude towards the way English is taught in the Language Centre which, according to them, is unmotivating, conventional and not engaging. As an English teacher, the researchers believe that engaging and involving learning environments are vital. Therefore, the main objective of this study is to explore the learning experience as a social interaction within Self-Organized Learning Environments (SOLEs). The aim is not to measure the effectiveness of SOLEs in English classes but to explore whether and how SOLEs affect the learning experience of English in Future College (pseudonym) in Oman, as an ecological exploration of enhancement rather than an outcomes-based intervention. 
One example of educators' trials to improve learning environments is known as Self-Organized Learning Environments (SOLEs) that were designed originally by Sugata Mitra after his experiments known as "The Hole in the Wall' that took place in India between 1999 and 2004 and refer to placing computers for children's free use [17]. Those "Hole in the Wall" experiences were later refined, moved inside school buildings and called SOLEs (ibid). A SOLE refers to a type of learning environments in which learners self-organise in groups of approximately four students and are provided with a computer connected to the Internet. The teacher's role is to facilitate a conceptually big and challenging question that is hard or impossible to be answered without the use of the Internet and has no right or wrong answer. Teachers then ask learners to search for answers to the given question on the Internet. A SOLE as an approach provides more room and freedom for learners, since they are allowed to talk to anyone, whether in their groups or not, they are allowed to walk around and to change groups. At the end of the session, they are asked to give an answer to the challenging question [17]. According to Mitra, 'Communication and collaboration are therefore key features of a SOLE' [17] (p. 232).

In theory, SOLEs appear to be able to offer much to enhance EFL learning environments and so suggest that students would benefit from such environments. In our view, SOLEs seem to be compatible with two important assumptions in learning which are 'people learn more by doing things themselves rather by being told about them' and 'learners are intelligent, fullyfunctioning humans, not simply receptacles for passed-on knowledge' [19] (p. 4). Although the evidence for how SOLEs work is incomplete and sparse, Mitra et al [17] claim to demonstrate that in SOLEs, students:

- Gain confidence

- Become capable of dealing with big challenging questions

- Learn things ahead of their age

- Retain knowledge longer

- Enjoy learning together, and individually

- Improve their computer and English language skills

It should be highlighted that apart from mentioning that SOLEs claim to help to improve English skills [17], there is no study that uniquely explores a SOLE as an English language learning environment. Research informs what works [15], therefore, SOLEs has to be scrutinized in order to prove or reject the above claims. Therefore, the main aim of this paper is:

To investigate whether SOLE pedagogy is able to positively influence Omani EFL college students' learning beliefs and behaviours. The research enables this through answering the research question: How do SOLEs impact students and their learning?

In sum then, a SOLE is a friendly unthreatening environment that promises a great deal, as articulated by Scrivener [19]. Nevertheless, it cannot be taken for granted that interaction in a SOLE is fruitful without proper rigorous research particularly in the case of SOLEs, on the basis that they have captured the public and popular interest in relation to a supposed revolutionary new way of teaching and reaching mass groups of pupils who may have hitherto been excluded for many and complex reasons [15]. Also, SOLE pedagogy is relatively new, and new methods of teaching and learning without systematic investigation may be considered questionable at best and dangerous at worst [15]. In sum, this study aimed to add to the literature of SOLEs by investigating the experience of SOLEs to inquire how such an approach affects students' experience of learning English. It is the first study to explore the use of SOLEs in teaching and learning English as a foreign language in the Omani context and thus offers unique educational insights.

\section{Literature review}

\subsection{Autonomy: definition, importance and significance in SOLEs}

Little [8] (p. 81) defines autonomy as 'a matter of the learner's psychological relation to the process and content of learning'. According to him, learners should be able to make decisions on their own learning and be able to take independent action that is congruent with their preferences, learning styles and strategies. Thanasoulas [9] (p. 47) also asserts that autonomous learners should take greater responsibility for their learning but he also emphasizes that the teacher's role is crucial and not redundant as teachers 'adapt resources, materials, and methods to the learners' needs and even abandon all this if need be'. Benson [2] provide some examples of autonomy such as when learners learn on their own, when learning takes place in self-directed environments and when learners are given the right to give directions for the learning process. Thanasoulas [9] believes that autonomy is in harmony with constructivism, as autonomous learners are active agents who construct their own learning through generating ideas, setting goals and linking new knowledge with existing knowledge, rather than receiving content from teachers.

Since its first emergence in the language teaching and learning field in 1971 [2], autonomy has been widely discussed in the EFL literature. Little [8] shed light on some characteristics of autonomous learners that include enthusiasm to learn, being active, acceptance of responsibility for their own learning, taking the initiative and being able to constantly 
evaluate and improve their learning strategy preferences. In the same vein, Harmer [15] asserts that students can become autonomous learners when they are able to make decisions on their best learning strategies. One way to help students become autonomous is to provide them with technology in which they can adapt and personalize their learning [2]. There are many other empirical studies focusing on ways of fostering learners' autonomy which cannot be mentioned here because they are beyond the scope of this work. However, almost all studies agree on the importance of fostering learners' autonomy and this is supported by the European Language Portfolio among the objectives of which is "to promote learner autonomy" [7] (p. 3). Thus, how to foster language learners' autonomy? Benson [2] in his book 'Teaching and Researching Autonomy in Language Learning' provides an answer to this question. He believes that learner autonomy can be fostered if students are given independent interaction with learning materials and technology, if they are given the chance to evaluate and control both the learning process and curriculum, if the importance of their behavioural changes is stressed and if they are supported by teachers to foster their autonomy.

Current SOLE methodology includes most of the suggested ways mentioned above that help to increase autonomy in classes. For example, in a SOLE, students choose what to do and think, they are able to make decisions, are responsible for their learning, learn on their own, learn in a self-directed environment and are able to choose their preferred learning strategies. However, this assumption must be empirically explored and studied.

Students in this project were given independent interaction in the learning environment, their input and evaluation of both the process and curriculum were considered and their decisions of learning styles and strategy preferences were taken into consideration. All these privileges given to students were scrutinized and, as a result, provided the researchers with a fuller picture that assisted in an indepth understanding of the influence of a SOLE on student autonomy as shown in the finding and discussion sections.

\subsection{Learner motivation: definition and importance in one's learning journey}

Motivation in the EFL literature is viewed as a core essential element that can facilitate learning in general and a new language learning in specific [12]. Moskovsky et al. [6] also assert that in the language learning field there is a lack of consensus among scholars in almost all issues except for the importance of motivation. Gardner [12] defines motivation as the love, desire and positive attitude towards acquiring and learning a new language. According to Higgins et al. [11], there are two factors that affect one's hopes and motivations. One is the ideal self which is considered internal motivation and refers to what one wishes to achieve and become. Another is the out-to self which is considered external and refers to the desire to meet the expectations of other people. It has been revealed that students' motivation decreases as they mature [16]. Lepper et al. [16] examined the relationship between intrinsic and extrinsic motivation and academic attainment. They used a sample that is ethnically different and from grade 3 to 8. Their findings revealed that there is a significant decrease in motivation as students grow up. Moskovsky et al. [6] found in their experimental study in which they asked teachers to use some preidentified motivational strategies for experimental groups but not for control ones. Their findings revealed very strong and compelling evidence that teachers' motivational behaviour strongly and positively affect learners' motivation. One of this project's main goals was to explore the influence of SOLEs on motivation since it is also claimed that initial observation shows that children find SOLEs more interesting and engaging compared to traditional education [17].

\section{Research Methodology}

This paper is part of a participatory action research project conducted to improve learning environments in the principal researcher's higher education context. Action research was originally coined by American psychologist Kurt Lewin in the 1940s, the term Action Research broadly refers to an applied form of social research that overtly aims at improving the social situation under study while simultaneously generating knowledge about it. [13] (p.103). Lewin contends that action research does not merely generate information but more importantly it is used to assess an intervention [13]. Another definition coined by Kemmis [10] is that action research is identification of strategies of planned action which are implemented, and then systematically submitted to observation, reflection and change'. Tripp [10] (p. 446) prefers to define action research as 'a form of action inquiry that employs recognised research techniques to inform the action taken to improve practice'. It is a cyclical process in which an intervention is tried out and then evaluated. Critically, in Participatory Action Research, the participants themselves are involved in decision making and evaluation. After evaluation, it is re-implemented again and re-evaluated again as well. This process should be repeated until obtaining the desired improvement and change. Action research follows a cycle that is followed in any basic action inquiry in which 'one plans, implements, describes, and 
evaluates an improving change to one's practice' [10] (p. 444), as shown in Figure 1 below.

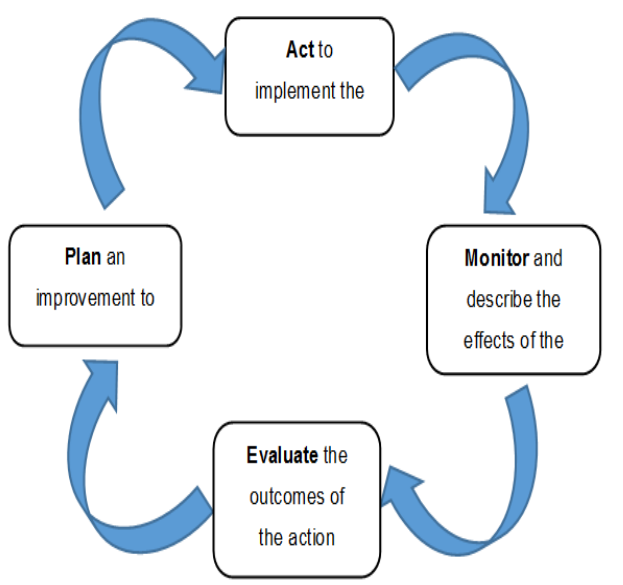

Figure 1. Representation of basic action inquiry cycle

Action research differs from other forms of action inquiry because 'action research, as a form of action inquiry, is an ongoing, repetitive process in which what is achieved in each cycle provides the starting point for further improvement in the next' [10] (p. 452). The table below represents the exact detailed steps conducted in action research after doing the reconnaissance.

Table 1. Representation of action research cycle

\begin{tabular}{|l|l|l|}
\hline & \multicolumn{2}{|l|}{ Action Taken in the Field of } \\
\hline Action sequence & Practice & Inquiry \\
\hline Planning of & $\begin{array}{l}\text { A change to } \\
\text { practice }\end{array}$ & $\begin{array}{l}\text { The evaluation of results of } \\
\text { the change to practice }\end{array}$ \\
\hline Implementation of & $\begin{array}{l}\text { The change to } \\
\text { practice }\end{array}$ & $\begin{array}{l}\text { Data production, analysis } \\
\text { and reporting }\end{array}$ \\
\hline Evaluation Of & $\begin{array}{l}\text { A) The change to practice } \\
\text { and; } \\
\text { B) The action inquiry } \\
\text { process }\end{array}$ \\
\hline
\end{tabular}

Source: extracted from Tripp (2005).

The researchers decided to adopt the action research design for its merits and because it is the most effective and suitable design for the ideas and questions in this research. One reason for adopting the action research design is that the researchers aimed to bring about a change in one specific context, as Tripp et al. [10], for example, emphasise, action research is a very effective design when the researcher aims at both generating knowledge and making improvements to current practices.

\subsection{Research context}

This study was conducted in an English language Centre that belongs to Future College which is located in the north of Oman and is part of the higher education system in Oman. The English Language Centre offers extensive English education to students in four different levels starting from level one to level four. This study was planned and designed after a reconnaissance that was carried out by the principal researcher earlier and by which it was found that students' attitudes towards the current English learning environments is mostly very negative. Those students spend twelve years in Omani schools studying all subjects in Arabic which is the medium of instructions and English is taught as a wholly academic rather than practical or living subject. Later, when they join higher education institutions like the college where this study took place, some of them find it difficult to adjust to the English only classes. This is due to their poor levels of English and their negative attitudes towards traditional learning environments which consist mostly of didactic and highly uninvolving environments and pedagogic practices, such as unmovable rows of chairs and tables, all facing one direction and without the possibility of conversation and dialogue. After the reconnaissance, the researchers decided to introduce SOLEs as a new English language environment in order to explore its impacts on students. It is worth highlighting that this paper is only a part of a larger project that also explores students' perceptions and experiences.

\subsection{Sampling procedures}

Multistage cluster sampling which includes random sampling as one procedure within it was adopted. Multistage cluster sampling is good when there are clusters in the population as in the case of this research. Cluster sampling is 'sampling of entire natural groups rather than individuals' [4] (p. 90). The research sample was recruited from the English foundation year (year one), male and female students aged 17-18 in the English Language Centre at the college. The sample consisted of 12 male students and 10 female students. The sample recruited is considered a probability sample which is believed to be representative of the whole population [3]. Two 
sessions a week were conducted with participants by the main researcher using SOLEs approach.

\subsection{Conduct of the study}

The process of conducting this study includes the following steps of Participatory Action Research, however, the last step is not explored in this paper:

- $\quad$ Planning of SOLE lessons.

- Implementing them.

- Describing and monitoring the implementation using the research methods.

- Evaluation of what happened.

- Suggestions for improvement that will be implemented in cycle two.

It is worth mentioning that reflection is not mentioned as an independent phase of participatory action research because it should occur in each singlephase due to its significance and effectiveness [10]. After cycle one, cycle two started as 'action research... is an ongoing, repetitive process in which what is achieved in each cycle provides the starting point for further improvement in the next' [10] (p. 452). Cycle two followed the same steps adopted in cycle one but with the suggested changes. Again, after cycle two, the field researcher suggested the best final SOLE model that is believed to work well and suit the culture and background of the adult Omani EFL learners.

At the beginning, the principal researcher set up a SOLE centre in Future College to enable him to implement the intervention which is a core element of any participatory action research. It was difficult to find a suitable room that could be transformed to a SOLE centre as all rooms were occupied. After a few days, it was decided that a room attached to the main library would be used. However, when starting to arrange the environment, it was considered to be too small and that it would not serve the purpose of this research. Finally, permission to use the resource centre which is a big room with round tables, computers, a white board, a projector and one big glass wall was obtained. The glass wall enables people outside to see what is happening inside which is preferred in setting up any SOLE centre. After allocation of the room, the process of setting up a SOLE centre started. Setting up a SOLE centre requires one computer per approximately four students, so in this case, six computers for the 22 participants were required in order to facilitate the collaborative work. It also requires one white board and large sheets of paper for students. Simply, a SOLE centre is a classroom with approximately five computers, four chairs or a u-shaped sofa in front of each computer, one white board and large sheets of paper. This room had everything needed except for large sheets of paper which the main researcher provided. The college administration assigned one technician to help in setting up the SOLE centre; the principal researcher and the technician worked together and prepared everything for students in the SOLE centre which was used for the full period of the study which was five months between September 2017 and January 2018.

After that, the sessions were planned, all materials were prepared, and then cycle one was started by conducting two weekly sessions (one hour each) with the participants using SOLEs as a pedagogical approach. These were the planning and implementation stages of PAR. The main researcher was knowledgeable in terms of conducting SOLE sessions having attended a module called 'The Future of Learning' which is taught by Mitra and focuses largely on SOLEs. In addition to that, instructions found in the SOLE toolkit were followed as shown in Figure 2.

The topic of each lesson was chosen from the same syllabus used for students in level four. The researchers together generated big questions for each lesson that covered the whole topic. Big questions in the SOLE context refer to questions that do not have direct answers and involve students working collaboratively, arguing, searching, synthesizing and evaluating information found while searching on the Internet. Big questions used in a SOLE are unique as they are the ones that provoke research, debate and critical thinking.

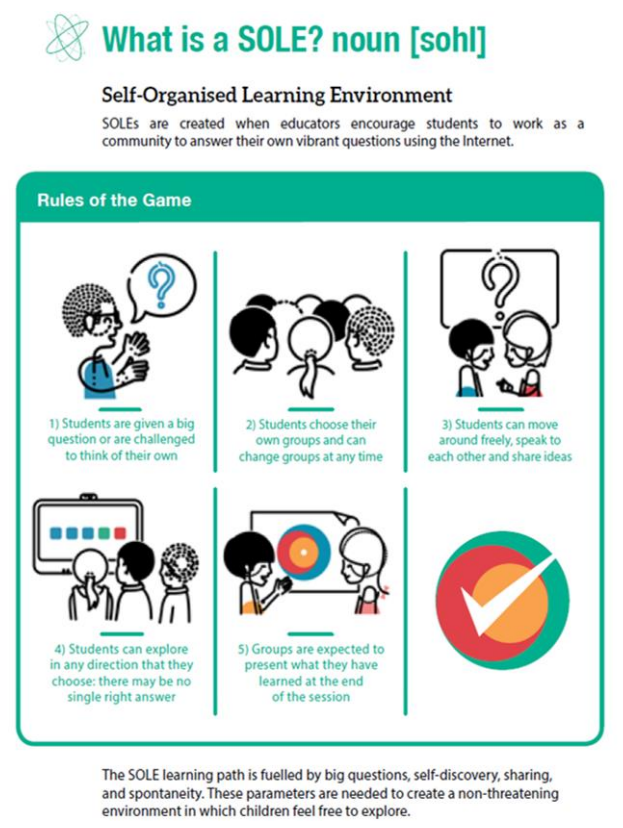

Figure 2. Steps of conducting a SOLE lesson

They are more concerned with the skills that lead to finding the rigorous reliable information and not the 
right answer. Big questions should be above the level of learners and have no easy direct answers so they stimulate collaborative work that always leads to collective answers and results in deep critical conversations among students [17]. Furthermore, 'a good big question will connect more than one subject area: What is an insect? For instance, does not touch as many different subjects as what would happen to the Earth if all insects disappeared?'. Therefore, the main researcher carefully went through current level four syllabus used in the English Language centre where the study took place. He generated questions that have the qualities of big questions explained above and made sure that those generated questions would serve the purpose of the study. Those big questions were presented to the original SOLE designer for approval. Completing these tasks is part of the planning stage of PAR which comes right after the reconnaissance stage. Some examples of big questions raised are the following:

- How does our gender affect our identity? This question was generated to cover unit one in the listening and speaking book.

- What would happen to the Earth if all primates were extinct? This question was generated to cover unit one in the reading and writing book.

- How would our life be without the discovery of DNA? This question was generated to cover unit two in the reading and writing book.

- What would happen if migration was banned? This question was generated to cover unit four in the listening and speaking book.

- How do you think natural hazards would affect our world in the next thirty years? This question was generated to cover unit four in the reading and writing book.

These are just examples of some of the big questions that were raised during the SOLE sessions in cycle one. After the planning stage which included planning SOLE sessions, the implementation stage of PAR started by conducting SOLE sessions, subsequently the involvement of participants in this study began and they remained involved until the end of cycle two. Each session started by giving students a big question and clarifying any unclear aspects of it for five minutes. Then students were asked to search for around forty minutes and at the end they were given approximately fifteen minutes to present their findings in the SOLE toolkit. During each session, the teacher left students unsupervised for some time as suggested by Mitra [17]. As part of the evaluation stage of PAR, while present in the SOLE, the main researcher took notes of everything happening including notes about participants, events and conversations which are crucial for any PAR research. Participants were also asked to reflect after each lesson on the learning experience in their diaries, semi-structured interviews were conducted with each participant one week before the end of cycle one and two focused group interviews were conducted at the end of cycle one. All these methods were used so that participants and the researchers could reflect immediately on the experience. They are also used, as mentioned above, for the evaluation stage of PAR in order to evaluate the intervention which is SOLEs and make any necessary changes for cycle two.

At the end, the researchers reflected and evaluated the whole experience. Participants' diaries, semistructured interviews, field notes and focus group data throughout cycle one were also analysed as it was an ongoing process. The rich data that were collected helped the researchers to make decisions about changes to be made especially those suggested by participants and stressed a lot in students' diaries, interviews, focus interviews and the researchers' field notes and evaluation; it is very important in PAR to involve participants throughout the research.

\subsection{Methods}

Four research methods were used to collect data which are semi-structured interviews, focus groups, diaries and the researcher field notes. It is worth mentioning that this is a purely qualitative study so using four methods ensure the trustworthy and authentic of the study as emphasized by Guba and Lincoln [cited in 10] who assert that in order for any qualitative study to be rigorous, it has to be both trustworthy and authentic.

\subsection{Analysis}

Thematic analysis was used for this study to analyse data stemming from diaries, semi-structured interviews, focus groups and the researcher's field notes. 'Thematic analysis is a method for identifying, analysing and reporting patterns (themes) within data' [20] (p. 79). The thematic analysis approach is very much used and acknowledged in qualitative research studies, it is seen and considered to be an essential foundational analytic tool [20]. The researchers adopted thematic analysis because it is considered essential and effective due to its flexibility that leads to not only rich deep data analysis but also a rigorous one (ibid). Not only that but it is also 'a constructionist method, which examines the ways in which events, realities, meanings, experiences and so on are the effects of a range of discourses operating within society' (ibid, p. 81). Additionally, thematic analysis is also important for qualitative researchers because it helps to theorize participants' experience within the 
studied sociocultural context and not simply focus on their psychologies (ibid).

\section{Findings}

\subsection{Students' autonomy increases}

The findings of this study revealed that students' autonomy increases in SOLEs. This theme was highlighted in the researchers' reflections and was confirmed by the participants. It was observed by the researcher and confirmed by participants that students were happy for the room provided by this environment to choose what to do and how. This environment helped to create autonomous learners. Participants mentioned many tasks that they completed in according with their own decision making. They mentioned that they were also able to express their opinions freely without limitations. The principal researcher noticed that students demonstrated autonomy in many actions in the SOLE environment. They chose which website to surf, how to tackle each task, how to set goals, who to consult and many other autonomy-generated decisions. Testimonies that demonstrate and confirm autonomy are detailed below.

Students highlighted that this environment gives them the room to complete activities the way they like, that is it gives them more freedom to tackle tasks according to their preference:

We do things that we like and the way we like (S2, students' interviews).

This way of learning gave the freedom for students to say their opinions whatever they are (S4, students' interviews).

Here students have more room to use their skills. Here students have options. Here students can choose any way to reach the answer (S20, focus groups).

The field researcher also noticed that SOLEs led to students becoming more autonomous in their learning as they started doing tasks the way they like and according to their own decision making:

Some students said it is good that we search in any website, it is our choice (the research field notes).

Students discussed how to search and do the task according to their preference (the research field notes).

\subsection{The approach in SOLEs motivates students}

Findings also showed that the approach of SOLEs motivates students. SOLEs motivate students to learn and work hard. Thirteen students mentioned that they felt motivated due to the availability of the Internet, social media, the reinforcement they receive, the uniqueness of SOLEs, the fun within SOLEs and additional reasons as shown in the extracts below:

This environment has motivated me to learn via the internet and social media. It has given us the motivation to be better in using programs (S3, students' interviews).

It Motivates students to like English language. This environment gives motivation for students to improve English skills, improve this language, and acquire new skills. It motivates us (S10, students' interviews). This environment motivates us and changed the routine of our education (S8, focus groups).

This environment increased my desire to learn because we learn in groups (S14, focus groups).

I like searching so Ifelt motivated by this environment (S15, focus groups).

I think this environment motivates students to study hard and to attain high marks (S4, students' diaries). Some students in all groups said 'let's work hard let's search in many websites'. This shows that they are very much motivated (the research field notes).

\section{Discussion}

\subsection{Students' autonomy increases}

The first theme which is students' autonomy increases was highlighted in the researchers' reflections and was confirmed by the participants. The researchers found that SOLEs help students to choose what to do and how, this finding was confirmed by some students, such as S2 who stated that 'we do things that we like and the way we like'. Students in this research made choices in how to answer questions, how to divide roles, how to present their outcomes and how to learn. This environment helped to create autonomous learners which is very important, as Thanasoulas [9] asserts, autonomous learners should take greater responsibility for their learning. Participants mentioned that they have more opportunities in SOLEs to make decisions. S20, for example, held that 'here students have more room to use their skills. Here students have options. Here students can choose any way to obtain the answer'. Giving students the opportunity to carry out some tasks according to their preferences is very important, this is in accordance with Little [8] who asserts that learners should be able to make decisions in terms of their own learning and to be able to take independent action that is congruent with their preferences, learning style and strategies. Some students in this study felt for the first time in their lives that they could control their own learning and make decisions during classes. 'Transferring some level of responsibility for learning to students...was in fact exceedingly motivating' [18] (p. 49). It was also emphasised that students felt that they could express their opinions freely without limitations. This is highlighted by S4 
who said that 'this way of learning gives students the freedom to state their opinions whatever they are'. This reflected positively on their attitude and emotional state towards learning English as evaluated and confirmed by the researchers. This is in congruence with the assertion by Little [8] (p. 81) that autonomy is linked to 'learner's psychological relation to the process and content of learning'.

The researchers noticed that students were autonomous in many tasks they performed. They chose which website to surf, how to tackle each task, how to set goals, who to consult and many other decisions. The field researcher noted all of these facts in his notes, writing 'some students said it is good that we search any website, it is our choice' and 'students discussed how to search and perform the task according to their preference'. This freedom given to students to choose which strategy to adopt is important, Harmer [15] asserts that students can become autonomous learners when they are able to make decisions on their best learning strategies. One way to help students become autonomous is to provide them with technology [2] and SOLEs provide computers connected to the Internet all the time which, according to this research, significantly assisted in helping to create autonomous learners. Computers formed a main factor that led to autonomy as it is a tool that provides students with a significant amount of freedom. It is their choice which website they surf and which articles and information they research. It is also their choice whether to use PowerPoint or another method to present their findings, all are accepted in SOLEs. Studies on autonomy agree on the importance of fostering learners' autonomy and this is supported by the European Language Portfolio among the objectives of it is "to promote learner autonomy" [7] (p. 3). Benson [2] in his book 'Teaching and Researching Autonomy in Language Learning' provides techniques to create autonomous learners. He believes that learner autonomy can be fostered if students are given independent interaction with learning materials and technology, if the importance of their behavioural changes is stressed and if they are supported by teachers in fostering their autonomy. SOLEs were found to support students in all these ways and, as a result, enhance student autonomy. Interaction with learning materials and technology is high in SOLEs and students' behavioural changes linked to their improvement in terms of personality and knowledge is acknowledged in this environment.

\subsection{The approach of SOLEs motivates students}

Motivation in the EFL literature is viewed as a core and essential element that can facilitate learning in general and new language learning in specific [12]. Moskovsky et al. [6] also assert that in the language learning field there is a lack of consensus among scholars on almost all issues except for the importance of motivation. Gardner [12] defines motivation as the love, desire and positive attitude towards acquiring and learning a new language. SOLEs have been found to motivate students to learn and work hard in this study; thirteen students mentioned that they felt motivated for various reasons. Several students felt motivated due to the availability of the Internet and social media, as S3 stated 'this environment has motivated me to learn via the Internet and social media. It has given us the motivation to be better in using programs'. Likewise, S15 stated that 'I like searching so I felt motivated by this environment'. This study stresses that learning environments that meet students' expectations and, most importantly, meet the requirements of their era help to motivate them. Students felt that this environment belonged to them, it is contemporary and suitable for their era. The researchers noticed the impact of SOLEs on students' motivation in many sessions, and noted that 'Some students in all groups said 'let's work hard, let's search many websites'. This indicates a high level of motivation. Additionally, improvement in their use and level of English proved to be motivating factors, as evidenced by S10 'SOLEs motivate students to like English language. This environment provides motivation for students to improve their English skills, improve this language, and acquire new skills. It motivates us'. In the same way, S4 said that 'I think this environment motivates students to study hard and to attain high marks'. Watt [14] argues that there are non-cognitive factors that affect students' motivation including the learning environment. This research confirms that SOLEs as learning environments function well in terms of motivating students. This finding is corroborated by the work of Ellis et al. [5] (p. 9) who found that students 'are being motivated in this process by the freedom to learn which SOLEs offer'. Therefore, students 'exhibited greater motivation' (ibid, p. 10). The teacher who trialled SOLEs in their study also expressed that 'the SOLE method appeared to energise the students' (ibid, p. 10). S8 who felt motivated due to the nature of SOLEs which breaks students' routines stated that 'this environment motivates us and changes the routine of our education'. Many students in this study felt motivated due to the element of enjoyment in SOLEs that is different from all of the routine classes to which they are accustomed. Others felt motivated by the group work which is a main pillar of SOLEs, as S14 stated that "this environment increased my desire to learn because we learn in groups'. The researchers found that group work leads to confidence and this confidence leads to motivation. It has been found that students' motivation decreases as they mature $[16 ; 14]$. Therefore, the ability of SOLEs to motivate adults in this study is a significant element of interest for this approach, since it helps to retain motivation 
and re-motivate older students. This study confirmed the motivational element of SOLEs that was revealed by the previous literature on this learning environment, it was highlighted that initial observation shows that children find SOLEs more interesting and engaging compared to traditional education [17]. Rix and McElwee [18] (p. 40) also found that 'initial enthusiasm...showed a move away from the passivity and apathy'. The validity of the results of this study is ensured as many tools were used, because the researchers explored the change in students' motivation during the period of a complete academic semester and also from different aspects. Moreover, these results highlight the factors that are present in SOLEs and which act as motivational drivers.

\section{Conclusion}

This paper aimed to explore SOLEs impacts on students. The researchers offered SOLEs as an intervention and then explored students' experiences in learning English using Self-Organized Learning Environments. SOLEs were chosen due to their supposed qualities emphasized by Mitra et al. (2016). They demonstrate that in SOLEs, students gain confidence, become capable, learn information ahead of their age, and enjoy activities.

The SOLE approach was implemented during the foundation year due to the importance of this pedagogical stage as it is the year in which students learn English intensively and prepare for their future specialization the following year. The objective that guided this research: to investigate whether SOLE pedagogy is able to positively influence Omani EFL college students.

This problem was addressed using participatory action research (PAR) because it helps researchers in both generating new knowledge and improving existing practices and situations [10]. Furthermore, action research allows researchers to act while researching by the use of interventions, which in this study were SOLEs. Another advantage of utilizing action research is that it is both participatory and collaborative [10], which means that the thinking, reflection, decisions and planning are all collective. In this study, the results of analyses were achieved after the analysis of collective views, reflections and evaluations by both the researchers and participants. Results revealed that in this study, students' autonomy increases, and approach of SOLEs motivates students. The researchers believe that future studies should examine SOLEs influence on students' attainment through appropriate mixed methodology studies.

\section{Acknowledgment}

The authors would like to thank the government of Oman for their sponsorship.

\section{References}

[1] Al-Mahrooqi, R., and C. Denman, English Education in Oman Current Scenarios and Future Trajectories. Springer, 2018.

[2] Benson, P., Teaching and researching: Autonomy in language learning. Harlow, England: Longman, 2001.

[3] Bryman, A., Social research methods. 3rd ED. New York: Oxford University press, 2008.

[4] Burns, R.B., Introduction to research methods. London: Sage publications, 2000.

[5] C. Ellis, A. Dyer and D. Thompson, (2014) "Riding tandem: an organic and collaborative approach to research in vocational education and training", Research in Learning Technology, 22, 2014, pp. 1-12.

[6] C. Moskovsky, F. Alrabai, S. Paolini and S. Ratcheva, "The effects of teachers' motivational strategies on learners' motivation: A controlled investigation of second language acquisition', Language Learning, 63(1), 2013, pp. 34-62.

[7] Council of Europe. European Language Portfolio (ELP): principles and guidelines. Strasbourg, France: Council of Europe, Language Policy Division, 2004.

[8] D. Little, “Autonomy in language learning", Teaching Modern Languages, 1990, pp. 81-87.

[9] D. Thanasoulas, "What is learner autonomy and how can it be fostered', The Internet TESL Journal, 6(11), 2000, pp. 37-48.

[10] D. Tripp, “Action research: a methodological introduction", Educacao e pesquisa, 31(3), 2005, pp. 443466.

[11] E.T. Higgins, R. Klein and T. Strauman, "Self-Concept Discrepancy Theory: A Psychological Model for Distinguishing among Different Aspects of Depression and Anxiety', Social Cognition, 3 (1), 1985, pp. 51-76.

[12] Gardner, R. C., Motivation and Second Language Acquisition: The Socio-Educational Model. New York: Peter Lang, 2010.

[13] Gilbert, N., Researching social life. 3rd ED. London: Sage, 2008.

[14] H.M. Watt, “A latent growth curve modeling approach using an accelerated longitudinal design: The ontogeny of boys' and girls' talent perceptions and intrinsic values 
through adolescence', Educational Research and Evaluation, 14(4), 2008, pp. 287-304.

[15] Harmer, J., The practice of English language teaching. 5th Ed. Pearson Education Ltd.UK, 2015.

[16] M.R. Lepper, J.H. Corpus and S.S. Iyengar, “Intrinsic and extrinsic motivational orientations in the classroom: age differences and academic correlates", Journal of educational psychology, 97(2), 2005, pp. 184.

[17] S. Mitra, S. Kulkarni and J. Stanfield, "Learning at the Edge of Chaos: Self-Organizing Systems in Education". Alternative Education, 2016, pp. 227-239.

[18] S. Rix, and S. McElwee, "What happens if students are asked to learn Geography content, specifically Population, through SOLE?', Other Education, 5(1), 2016, pp. 30-54.

[19] Scrivener, J., Learning teaching. Oxford: Heinemann, 1994.

[20] V. Braun and V. Clarke, V. "Using thematic analysis in psychology", Qualitative Research in Psychology, 3(2), 2006, pp.77-101 